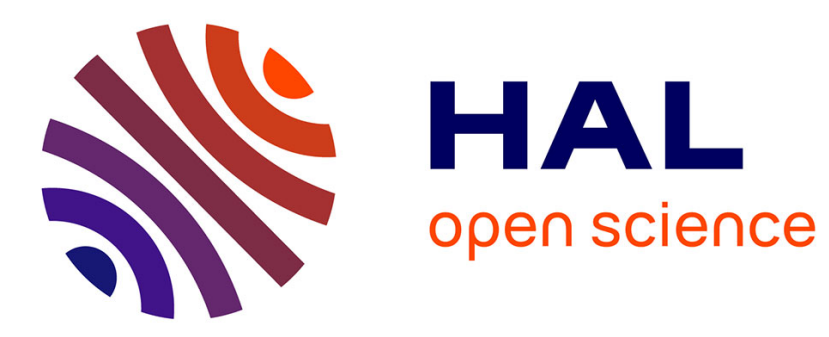

\title{
Observability analysis and sensor placement
}

\author{
Didier Maquin, Marie Luong, José Ragot
}

\section{To cite this version:}

Didier Maquin, Marie Luong, José Ragot. Observability analysis and sensor placement. IFAC/IMACS Symposium on Fault Detection, Supervision and Safety for Technical Processes, SAFEPROCESS'94, Jun 1994, Espoo, Finland. pp.754-759. hal-00302900

\section{HAL Id: hal-00302900 https://hal.science/hal-00302900}

Submitted on 17 Apr 2014

HAL is a multi-disciplinary open access archive for the deposit and dissemination of scientific research documents, whether they are published or not. The documents may come from teaching and research institutions in France or abroad, or from public or private research centers.
L'archive ouverte pluridisciplinaire HAL, est destinée au dépôt et à la diffusion de documents scientifiques de niveau recherche, publiés ou non, émanant des établissements d'enseignement et de recherche français ou étrangers, des laboratoires publics ou privés. 


\title{
OBSERVABILITY ANALYSIS AND SENSOR PLACEMENT
}

\author{
Didier MAQUIN, Marie LUONG and José RAGOT
}

Centre de Recherche en Automatique de Nancy - CNRS UA 821

2, avenue de la forêt de Haye - 54516 Vandouvre - France

Phone: (33) 83595959 Fax: (33) 83595644 E.mail: \{dmaquin, mluong, jragot\}@ensem.u-nancy.fr

\begin{abstract}
The quality of the state estimation of a system is strongly conditioned by the number of measurements obtained from this system. Here, this dependence is studied by specifying especially the influence of the number and the position of sensors as well as of their precision. When some variables are necessary for the control, the possibility of failure of some sensors is analysed from the calculation of the whole system reliability. The reciprocal problem is also presented: what is the measurement system allowing to respect some constraints on the observability, the redundancy and the reliability.
\end{abstract}

Key Words. Sensor placement; observability; redundancy; reliability; control system design; sensor failures; measurement system

\section{INTRODUCTION}

Nowadays, the conception of a measurement system is of fundamental importance. Indeed, the position of sensors and their number condition the possibility to observe a process, to estimate its state and consequently to determine the type of control to apply. Studying the observability of a system consists in answering the question: can we determine the value of variables of a system from a given set of measurements ? If the process is non observable, observable and non observable parts may however be determined. More generally, the posed problem is that one of diagnosis which consists in estimating the state of a system, deciding if it is normal or abnormal, judging the gravity of the abnormal state and with this appreciation deciding an action to be undertake in order to preserve the system functionalities. As we can quite imagine, this estimation is possible only under some conditions and especially requires to have a minimum amount of information that is to say a minimum amount of sensors; these sensors have to be judiciously placed in order to give an "image" of the totality of the process. Besides, the precision of collected information, closely linked to the precision of sensors and to their good functioning, conditions the precision of the diagnosis very largely. Finally, it is necessary to consider the realistic case in which initially provided sensors can fail. Consequently to these failures, the diagnosis of the state of the process can be performed only if variables measured by these faulty sensors can be estimated. Considering these remarks, one can assert that the choice of the instrumentation (number, position and characteristics of sensors) is an essential factor of the outcome of diagnosis. In the following, our purpose is limited to the study of system described by linear equations (for example, they can correspond to equations of matter or energy conservation), having concern for the simplicity, the generalisation to more complex models does not create major problems.

Many works on observability analysis of steady-state systems have already been published. Firsts of them have focused on the linear system study (Vaclaveck et al., 1969); afterwards, Stanley and Mah(1981) and Crowe et al. (1983) have largely contributed to develop this analysis. Algorithms of observability that have been developed are generally based on graph theory (Mah et al., 1976) or on a classification of variables from a projection matrix (Crowe, 1989), (Ragot et al., 1994). For systems described by linear-bilinear equations the first studies of observability are due to Vaclaveck and Loucka (1976), Romagnoli and Stephanopoulos (1980). Kretsovalis and Mah (1988) have then extended this study by using graph theory. Concurrently, Darouach (1986) has formalised the study of the observability of bilinear systems in the matrix form; the method employed is summarised to a sequential study of the observability of linear systems (Maquin et al., 1989). More recently, Ragot et al. (1990) proposed an algorithm based on the simultaneous analysis of linear and bilinear equations.

After the analysis phase, making in evidence not only redundancy but also the possible weaknesses of the measurement system, it is advisable to propose modifications of the existent sensor placements or even sensor addition. These modifications are studied considering precise objectives: to render observable a particular variable, to increase the degree of redundancy of another one, to enhance the precision of the estimation of a particularly useful variable for the control of the process, to tolerate sensor failures. Precisely, our purpose is to analyze the different criteria allowing to define the choice of the measurement system.

\section{SYSTEM STATE ESTIMATION}

The state estimation of a system is, in general, proceeded by data reconciliation with respect to the exact and known supposed model of the system. Indeed, measurements 
provided from inputs and outputs of the system do not verify its constraint equations. It is then advisable to correct these measurements, therefore to estimate probable values of the corresponding variables, so as to meet these equations. In what follows, we note:

$\mathrm{X}^{*}$ the actual value vector $\left(\in \mathrm{R}^{\mathrm{m}}\right)$

$\mathrm{X}$ the measurement vector $\left(\in \mathrm{R}^{\mathrm{m}}\right)$

$\hat{\mathrm{X}}$ the estimation vector $\left(\in \mathrm{R}^{\mathrm{m}}\right)$

A the constraint matrix $\left(\in \mathrm{R}^{\mathrm{n} \cdot \mathrm{m}}\right)$

$\mathrm{V}$ the variance-covariance matrix of measurement errors

Assuming that the measurement errors are zero-mean and considering the case where the variance-covariance matrix $\mathrm{V}$ is known, the maximum likelihood estimation $\hat{\mathrm{X}}$ is solution of the optimization problem:

$\min \Phi=\frac{1}{2}\|\mathrm{X}-\hat{\mathrm{X}}\|_{\mathrm{V}^{-1}}^{2}$

subject to $\mathrm{A} \hat{\mathrm{X}}=0$

This very classic problem can be solved by different techniques. All, they give the same result with numerical errors caused by truncatures appearing in the different calculations:

$\hat{\mathrm{X}}=\mathrm{P} \mathrm{X}$

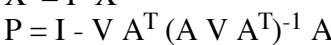

The variance-covariance matrix of $\hat{X}$ is given by:

$\hat{\mathrm{V}}=\mathrm{P} \mathrm{V}$

This expression provides the precision of estimations and therefore defines their confidence interval; as direct consequence, estimated confidence intervals of corrective terms of measurements can also be used to detect possible anomalies (Ragot et al., 1990).

Most of systems are partially observed only and the number of available measurements is in general much inferior than that of variables whose state is to be estimated. The estimation problem has therefore to be reexamined considering this constraint. The measurement vector is then linked to the actual value vector and the measurement error vector by:

$\mathrm{X}=\mathrm{HX}^{*}+\varepsilon$

The estimation $\hat{X}$ is solution of the optimization problem described by equation (1). The conditions of existence for this solution $\hat{\mathrm{X}}$ are closely related to the rank of the observability matrix $\left(\mathrm{H}^{\mathrm{T}} \mathrm{A}^{\mathrm{T}}\right)$. If this rank is equal to the dimension of $\mathrm{X}$ (Darouach, 1986), the system state is totally observable and its estimation can be written as:

$\hat{X}=\left(R-R A^{T}\left(A R A^{T}\right)^{-1} A R\right) H^{T} V^{-1} X$

$\mathrm{R}=\left(\mathrm{H}^{\mathrm{T}} \mathrm{V}^{-1} \mathrm{H}+\mathrm{A}^{\mathrm{T}} \mathrm{A}\right)^{-1}$

This expression shows that the estimation depends on the measurement system (number and placement of sensors through the matrix $\mathrm{H}$ ), the measurements themselves $\mathrm{X}$ and their precisions $\mathrm{V}$; it is necessary to specify this dependence so as to improve the quality of these estimations (see section 4).

\section{SYSTEM OBSERVABILITY}

In practice, it is interesting to test the observability condition directly from the network structure. Different solutions have been proposed among which that using the analysis of cycle matrix associated to the system graph which is simple and elegant to implement (Gomolka et al.., 1992); here, we remind the principle of this analysis.

\subsection{Cycle matrix of a system}

Let us consider the graph (Fig. 1) of a simple process, where the node ' 0 ' represents the environment one (for a system of matter transportation for instance, the environment node connects entry and exit flows of this system). One wishes to enumerate the observable variables of this system, more precisely to classify variables in deducible ones and redundant ones.

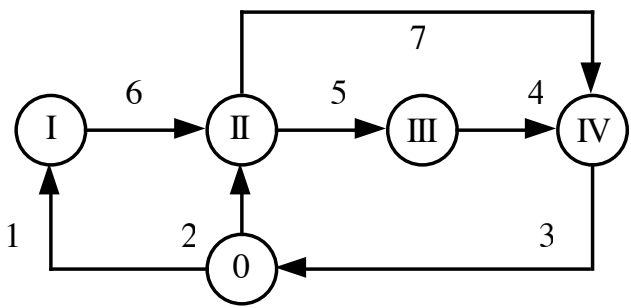

Fig. 1. Nodes and arcs of a graph

The fundamental cycle matrix CF of the fig. 2 is obtained from the fig. 1. In this matrix $\mathrm{CF}$, each column corresponds to an arc and each row to a fundamental cycle.

$\mathrm{CF}=\begin{aligned} & 1 \\ & 2 \\ & 3\end{aligned}\left[\begin{array}{lllllll|}1 & 2 & 3 & 4 & 5 & 6 & 7 \\ \hline . & 1 & 1 & 1 & 1 & . & . \\ 1 & 1 & . & . & . & 1 & . \\ . & 1 & 1 & . & . & . & 1\end{array}\right.$

Fig. 2. Fundamental cycle matrix

A mathematical operation performed on the fundamental cycle matrix $\mathrm{CF}$ allows all the cycles contained in a graph to be enumerate. By definition, a fundamental cycle matrix constitutes a basis of cycles. By making a logical xor between two rows of $\mathrm{CF}$ (which represent fundamental cycles), one can list all the cycles contained in a graph. For our example, we deduce the following cycle matrix $\mathrm{C}$ :

\begin{tabular}{|c|c|c|c|c|c|c|c|}
\hline & 1 & 2 & 3 & 4 & 5 & 6 & 7 \\
\hline 1 & 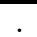 & 1 & 1 & 1 & 1 & 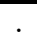 & . \\
\hline 2 & 1 & 1 & . & . & . & 1 & 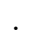 \\
\hline 3 & & 1 & 1 & . & & & 1 \\
\hline $\mathrm{C}=$ & 1 & . & 1 & 1 & 1 & 1 & . \\
\hline 5 & & . & . & 1 & 1 & . & 1 \\
\hline 6 & 1 & & 1 & & & 1 & 1 \\
\hline
\end{tabular}

Fig. 3. Matrix of all the cycles of the graph

\subsection{Observability analysis}

The previous cycles are constituted of arcs corresponding to measured or unmeasured variables. Depending on the number of measured variables per cycle, one can define the observability of all the system variables. The cyclebased observability study is realised through four elementary rules (Turbatte et al., 1993):

Rule I: a measured variable is estimable if, and only if, it only intervenes in cycles where two variables are measured at least.

Rule II: a measured variable is non estimable if, and only if, it belongs at least to a cycle where it is the alone measured variable.

Rule III: an unmeasured variable is deducible if, and only if, it only intervenes in cycles comprising at least a measured variable. 
Rule IV: an unmeasured variable is non deducible if, and only if, it belongs at least to a cycle where no variable is measured.

From process of the fig. 1, let us show the usage of these four rules. For instance, consider the following measured and unmeasured variable lists:

$\mathrm{L}_{\mathrm{m}}=\{1,2,3\} \quad \mathrm{L}_{\overline{\mathrm{m}}}=\{4,5,6,7\}$

Considering these lists, the application of observability rules to variables of the matrix $\mathrm{C}$ yields the next conclusions. Variables $\{1,2,3\}$ are measured and estimable. These variables intervene only in cycles where two variables are measured (rule I). The variable 6 is unmeasured but deducible; indeed, cycles to which it belongs contain all a measured variable at least (rule II). Variables $\{4,5,7\}$ are unmeasured and non deducible. These variables belong to the cycle 5 where all variables are unmeasured (rule IV).

It is important to note that changing the measured variable list (sensor addition, withdrawal or moving) is simple to take into account. Indeed, the preceding analysis is based on the enumeration of cycles which is carried out independently of measurements. Now, the conception of measurement system profit by this remark.

\subsection{Synthesis of a measurement system according to the observability concept}

The preceding analysis has allowed to enumerate deducible variables, redundant ones and those that cannot be estimated. When the "observability degree" of all the variables is judged unsatisfied, it is advisable to measure supplementary variables by placing a certain number of sensors judiciously. This choice can be made by analyzing the cycle matrix of the system. In a very general manner this latter can be put under the form:

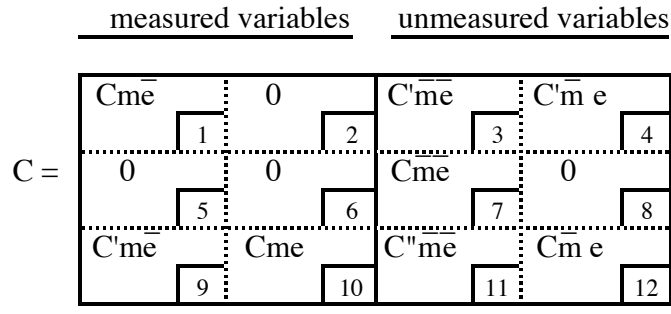

Fig. 4. General form of the cycle matrix

The above-mentioned decomposition yields the next classification of variables:

- Measured and non estimable variables are part of the bloc 1 (matrix Cme ). There is only a '1' by row in this submatrix (rule II).

- Estimable and measured variables are part of the submatrix 10 (matrix Cme). In this submatrix, there is at least two measured variables by row (rule I).

- Unmeasured and non deducible variables are ordered in the submatrix 7 (matrix $\mathrm{Cm}^{-\bar{e}}$ ). This submatrix intervenes in a "row" where no variable is measured; all the other submatrices are null (rule IV).

- Unmeasured and deducible variables intervene in submatrices 4 and 12 (matrices $C^{\prime} \bar{m}$ e and $C^{-} \bar{m}$ e). These submatrices are part of a "row" containing measured variable submatrices - submatrices 9 and 10 - (rule II).

Consider the situation where some variables a priori are measured (list $\mathrm{L}_{\mathrm{m}}$ ); different criteria can be envisaged to complete this list so as to increase the observability degree of the system. For example, one can wish that some variables become deducible, or that others become redundant; moreover, one can impose that some of these variables could not be measured.

As an example, consider the case where $\mathrm{L}_{\mathrm{m}}=\{1\}$ and where the variable 7 cannot be measured; one wishes to render observable all the variables of the system with respect to these constraints. In the cycle matrix of the fig. 3 , one observes that the definition of $\mathrm{L}_{\mathrm{m}}$ allows to enumerate 3 cycles (2, 4 and 6$)$ containing the measured variable 1 . Therefore, it is necessary that the remaining cycles (1, 3 and 5) contain each a measured variable at least in order to insure the observability of all the variables of the system. To obtain that and to minimise the number of supplementary sensors, a couple can be chosen among next ones:

$\{2,4\},\{2,5\},\{2,7\},\{3,4\}$

$\{3,5\},\{3,7\},\{4,5\},\{5,7\}$

Among these eight solutions, the choice will be able to be made according to a complementary criterion, for example of minimal cost, maximal reliability or desired estimation precision.

\section{ESTIMATION ANALYSIS}

The estimation procedure provides the state of the system through the measurements and their respective precisions. Though, it is very useful to complete this study by characterising the estimations by their sensitivity with respect to the parameters describing the process. As process equations a priori are supposed exact, the sensitivity study is here reduced to the influence of a modification of the measurements and their number.

\subsection{Sensitivity to a small variation of the measurement precision}

A variation $\Delta \mathrm{V}$ of the measurement variance induces a corresponding estimation variation $\Delta \hat{\mathrm{X}}$ whose expression is deduced from (2):

$\hat{\mathrm{X}}+\Delta \hat{\mathrm{X}}=\left(\mathrm{I}-(\mathrm{V}+\Delta \mathrm{V}) \mathrm{A}^{\mathrm{T}}\left(\mathrm{A}(\mathrm{V}+\Delta \mathrm{V}) \mathrm{A}^{\mathrm{T}}\right)^{-1} \mathrm{~A}\right) \mathrm{X}$

After making some tedious calculations and taking into account small variations such that $\Delta \mathrm{V} \ll \mathrm{V}$, the relationship (6) is then reduced to:

$\Delta \hat{\mathrm{X}}=\mathrm{P} \Delta \mathrm{VV}^{-1}(\hat{\mathrm{X}}-\mathrm{X})$

By noting $\mathrm{p}_{. \mathrm{i}}$ the $\mathrm{i}^{\text {th }}$ column of the projection matrix $\mathrm{P}, \mathrm{v}_{\mathrm{ii}}$ the $i^{\text {th }}$ diagonal term of $\mathrm{V}$ and $\mathrm{x}_{\mathrm{i}}$ the $\mathrm{i}^{\text {th }}$ component of $\mathrm{X}$, the vector $\Delta \hat{\mathrm{X}}$ can be written as:

$\Delta \hat{\mathrm{X}}=\sum_{\mathrm{i}=1}^{\mathrm{v}} \mathrm{p}_{. \mathrm{i}} \frac{\Delta \mathrm{v}_{\mathrm{ii}}}{\mathrm{v}_{\mathrm{ii}}}\left(\hat{\mathrm{x}}_{\mathrm{i}}-\mathrm{x}_{\mathrm{i}}\right)$

Thus, the vector of estimation sensitivity with respect to the variance of the $i^{\text {th }}$ measurement can be written:

$\mathrm{S}=\frac{\partial \hat{\mathrm{X}}}{\partial \mathrm{v}_{\mathrm{ii}}}=\mathrm{p}_{. \mathrm{i}} \frac{1}{\mathrm{v}_{\mathrm{ii}}}\left(\mathrm{x}_{\mathrm{i}}-\mathrm{x}_{\mathrm{i}}\right)$ 
In this calculation, one can avoid utilising the terms $\mathrm{p}_{\mathrm{i}}$ of the projection matrix by eliminating the matrix $\mathrm{P}$ by using (3). We obtain:

$\Delta \hat{\mathrm{X}}=\hat{\mathrm{V}} \mathrm{V}^{-1} \Delta \mathrm{V} \mathrm{V}^{-1}(\hat{\mathrm{X}}-\mathrm{X})$

By performing the different matrix products that appear in (11), coefficients of sensitivity can be expressed under the form:

$s_{i j}=\frac{\partial \hat{x}_{i}}{\partial v_{j j}}=\hat{v}_{j i} \frac{\hat{x}_{i}-x_{i}}{v_{i i}^{2}}$

Using this relationship, the ratio of estimation sensitivities of two variables $j$ and $k$ when the variance of the variable $i$ varies can be deduced:

$\frac{s_{i j}}{s_{i k}}=\frac{\hat{v}_{j i}}{\hat{v}_{k i}}$

Thus, when the measurement variance changes, all the estimations are modified; the estimation that undergoes the greatest variation is that whose variance is the most important. There, it concerns an intuitive result whose equation (12) gives the theoretical justification.

\subsection{Sensitivity with respect to any variation of the} measurement precision

To simplify this study, only one variance is modified of the quantity $\delta$ at a time (for example the $i^{\text {th }}$ ). Thus, we note:

$\Delta \mathrm{V}=\delta \mathrm{EE}^{\mathrm{T}}$

where all the components of the vector $\mathrm{E}$ are null except for the $\mathrm{i}^{\text {th }}$ which is equal to one. After modifying the measurement variance, estimations are always expressed by the relationship (6). Using the lemma of matrix inversion and with $\mathrm{K}=(\mathrm{A} \mathrm{V} \mathrm{A})^{\mathrm{T}}$ we obtain:

$\Delta \hat{\mathrm{X}}=\frac{\delta}{1+\delta E^{\mathrm{T}} \mathrm{A}^{\mathrm{T}} \mathrm{KAE}} \mathrm{PEE}^{\mathrm{T}} \mathrm{V}^{-1}(\hat{\mathrm{X}}-\mathrm{X})$

This relationship therefore quantifies the influence of a variation of the measurement variance on the state estimation of the system.

\section{ANALYSIS OF THE ESTIMATION PRECISION}

We propose to examine the influence of any amplitude variations of the measurement precision on the estimation variance. An interesting limit case occurs where this variation is of infinite amplitude; that case practically corresponds to suppressing the corresponding measurement and therefore to testing the influence of a measurement on the precision of estimations.

As previously, we modify one variance of the quantity $\delta$ at a time (the $\mathrm{i}^{\text {th }}$ for example). Thus, we note:

$\Delta \mathrm{V}=\delta \mathrm{EE}^{\mathrm{T}}$

where all the components of the vector $\mathrm{E}$ are null except for the $i^{\text {th }}$ which is equal to one. After modifying the measurement variance, estimation variance is still the following:

$\hat{\mathrm{V}}+\Delta \hat{\mathrm{V}}=(\mathrm{V}+\Delta \mathrm{V})\left(\mathrm{I}-\mathrm{A}^{\mathrm{T}}\left(\mathrm{A}(\mathrm{V}+\Delta \mathrm{V}) \mathrm{A}^{\mathrm{T}}\right)^{-1} \mathrm{~A}(\mathrm{~V}+\Delta \mathrm{V})\right)$
After usual simplification, we obtain:

$\Delta \hat{V}=\frac{\delta}{1+\delta E^{\mathrm{T}} \mathrm{A}^{\mathrm{T}} \mathrm{KAE}} \mathrm{PEE}^{\mathrm{T}} \mathrm{P}^{\mathrm{T}}$

This formula is not an approximation; it exactly expresses the influence of a modification $\delta$ of the measurement variance on the variance of estimations. In the particular case where this modification has a weak amplitude, we have:

$\Delta \hat{\mathrm{V}}=\delta \mathrm{PEE}^{\mathrm{T}} \mathrm{P}^{\mathrm{T}}$

Finally, a more elegant form can be obtained by using the variance of estimations:

$\hat{\mathrm{V}}=\mathrm{V}-\mathrm{VA}^{\mathrm{T}}\left(\mathrm{AVA}^{\mathrm{T}}\right)^{-1} \mathrm{~A} \mathrm{~V}$

From (19), the following expression is extracted:

$\mathrm{A}^{\mathrm{T}} \mathrm{KA}=\mathrm{V}^{-1}-\mathrm{V}^{-1} \hat{\mathrm{V}} \mathrm{V}^{-1}$

Thus (18) implies:

$\Delta \hat{V}=\frac{\delta}{1+\delta E^{T}\left(V^{-1}-V^{-1} \hat{V} V^{-1}\right) E} \hat{V} V^{-1} E E^{T} V^{-1} \hat{V}$

So, taking into account the expression of the vector $\mathrm{E}$, the general term $\Delta \hat{\mathrm{v}}_{\mathrm{pq}}$ can be written as:

$\Delta \hat{v}_{p q}=\frac{\delta}{v_{i i}^{2}+\delta\left(v_{i i}-\hat{v}_{\text {ii }}\right)} \stackrel{\hat{v}}{p i}_{\text {pi }} \stackrel{\wedge}{q i}$

Thus, the diagonal coefficients of this matrix have the following expression:

$\Delta \hat{\mathrm{v}}_{\mathrm{kk}}=\frac{\delta}{\mathrm{v}_{\mathrm{ii}}^{2}+\delta\left(\mathrm{v}_{\mathrm{ii}}-\hat{\mathrm{v}}_{\mathrm{ii}}\right)} \stackrel{\wedge}{\mathrm{v} k}_{\mathrm{kk}}^{2}$

and they represent the variation of the estimation variances according to a modification of amplitude $\delta$ of a measurement variance.

\subsection{Application to the suppression of sensors}

The particular case where the amplitude variation $\delta$ is infinite corresponds to an interesting situation. Since $\delta$ is directly related to the measurement variance, it is equivalent to consider that the precision of this measurement is equal to zero; the weight of this measure in the criterion of estimation therefore becomes null. Thus, with a roundabout manner which is simple to implement though, we can study the influence of the suppression of a measure on the variance of estimated variables.

Let us explain therefore the limit of (22), when $\delta$ tends to infinity:

$$
\Delta \hat{\mathrm{v}}_{\mathrm{pq}}=\frac{1}{\left(\mathrm{v}_{\mathrm{ii}}-\hat{\mathrm{v}}_{\mathrm{ii}}\right)} \hat{\mathrm{v}}_{\mathrm{pi}} \hat{\mathrm{v}}_{\mathrm{qi}}
$$

From this expression, one may deduce the terms related to the modification of the estimation variances:

$\Delta \hat{\mathrm{v}}_{\mathrm{kk}}=\frac{1}{\left(\mathrm{v}_{\mathrm{ii}}-\hat{\mathrm{v}}_{\mathrm{ii}}\right)} \stackrel{\mathrm{v}}{\mathrm{kk}}^{2}$

Especially, for the $i^{\text {th }}$ variable (whose measurement has a infinite variance), the modification of the variance of estimations is the following: 
$\Delta \hat{\mathrm{v}}_{\mathrm{ii}}=\frac{1}{\mathrm{v}_{\mathrm{ii}}-\hat{\mathrm{v}}_{\mathrm{ii}}} \hat{\mathrm{v}}_{\mathrm{ii}}^{2}$

that therefore corresponds to the new variance:

$\hat{\mathrm{v}}_{\mathrm{ii}}+\Delta \hat{\mathrm{v}}_{\mathrm{ii}}=\frac{\mathrm{v}_{\mathrm{ii}} \stackrel{\hat{\mathrm{v}}}{\mathrm{ii}}}{\mathrm{v}_{\mathrm{ii}}-\hat{\mathrm{v}}_{\mathrm{ii}}}$

The whole established relationships are useful to analyse the results of the data reconciliation. These relationships provide this analysis a way to appreciate the contribution of the measurements and their respective variances to the construction of their estimations and variances.

\subsection{Synthesis of the measurement system from the estimation precision}

The preceding study has shown the influence of the precision of measurements on that of their estimations. Conversely, one can seek to determine the measurement variance (therefore specify the corresponding sensor precision) to obtain a given variance of the estimations. Remind the expression of the estimation variance:

$\hat{\mathrm{V}}=\left(\mathrm{I}-\mathrm{V} \mathrm{A}^{\mathrm{T}}(\mathrm{A} \mathrm{V} \mathrm{A})^{-1} \mathrm{~A}\right) \mathrm{V}$

Knowing V, can we then determine $\hat{V}$ ? To solve this problem, notice first of all that $\hat{V}$ cannot be arbitrarily chosen. This matrix indeed might verify the constraint:

$\mathrm{A} \hat{\mathrm{V}}=0$

To exploit easily this constraint, let us use an equivalent form of A obtained by extraction of its regular part and let us partition correspondingly the matrix $\mathrm{V}$ (chosen diagonal) and $\hat{\mathrm{V}}$ :

$\mathrm{A}=\left(\begin{array}{ll}\mathrm{I} & \mathrm{N}\end{array}\right)$

$\mathrm{V}=\left(\begin{array}{cc}\mathrm{V}_{11} & 0 \\ 0 & \mathrm{~V}_{22}\end{array}\right) \quad \hat{V}=\left(\begin{array}{ll}\hat{V}_{11} & \hat{V}_{12} \\ \hat{V}_{21} & \hat{V}_{22}\end{array}\right)$

By expressing (28) from (30) and (31), we obtain:

$\hat{\mathrm{V}}_{11}=\mathrm{V}_{11}-\mathrm{V}_{11} \mathrm{H} \mathrm{V}_{11}$

$\hat{\mathrm{V}}_{12}=-\mathrm{V}_{11} \mathrm{H} \mathrm{N} \mathrm{V} 22$

$\hat{\mathrm{V}}_{21}=-\mathrm{V}_{22} \mathrm{~N}^{\mathrm{T}} \mathrm{H} \mathrm{V}_{11}$

$\hat{V}_{22}=V_{22}-V_{22} N^{T} H V_{22}$

with:

$\mathrm{H}=\left(\mathrm{V}_{11}+\mathrm{N} \mathrm{V}_{22} \mathrm{~N}^{\mathrm{T}}\right)^{-1}=\mathrm{H}\left(\mathrm{V}_{11}, \mathrm{~V}_{22}\right)$

Then, the equations (32a) and (32d) that constitute a non linear system with respect to $\mathrm{V}_{11}$ and $\mathrm{V}_{22}$ can be solved by using, for example, an algorithm with direct iterations:

$\mathrm{V}_{11}^{\mathrm{k}+1}=\hat{\mathrm{V}}_{11}+\mathrm{V}_{11}^{\mathrm{k}} \mathrm{H}\left(\mathrm{V}_{11}^{\mathrm{k}}, \mathrm{V}_{22}^{\mathrm{k}}\right) \mathrm{V}_{11}^{\mathrm{k}}$

$\mathrm{V}_{22}^{\mathrm{k}+1}=\hat{\mathrm{V}}_{22}+\mathrm{V}_{11}^{\mathrm{k}} \mathrm{N}^{\mathrm{T}} \mathrm{H}\left(\mathrm{V}_{11}^{\mathrm{k}}, \mathrm{V}_{22}^{\mathrm{k}}\right) \mathrm{N} \mathrm{V}_{22}^{\mathrm{k}}$

Practically, the values of $\hat{V}_{11}$ and $\hat{V}_{22}$ are given and the stable solution of (33) is searched. The equations (32b) and (32c) then give the estimation variances that have not been able to be imposed.

\section{RELIABILITY OF A MEASUREMENT SYSTEM}

For a real process, the calculation of estimations as well as that of their sensitivity that has been presented supposes that the measurements are available that is to say that sensors are able to deliver them. In some cases, the failure of a sensor does not involves necessarily the impossibility to calculate the estimation; this notion was specified in the section 2 by defining the condition to meet this and giving the expression of the estimation according to available measurements. If this condition of observability is not satisfied, the complete state estimation of the system is not possible. These situations have to be specified and can be characterised by an indication of reliability of the measurement system. A second motivation of this calculation of reliability stem from the necessity to have a certain number of measured variables allowing a security for the controlling of the process. Necessary variables for this control are in general directly measured, but it is then necessary to insure that a variable remains deducible if the associated sensor becomes failing. The situation complicates with the number of failures one can accept while preserving the observability of this necessary variable list for the control of the system. Let us present now the mean to evaluate the reliability of a measurement system.

By definition, the reliability of a sensor is the probability $\mathrm{r}(\mathrm{t})$ that at the instant $\mathrm{t}$, there is no failure knowing that no failure had occurred at the instant $t=0$. This probability may be expressed analytically by using, for example, the law of Poisson:

$r(t)=\exp (-\lambda t)$

where $\lambda$ is a constant representing the rate of sensor failure. As an extension, one may define the reliability of a system constituted of $\mathrm{n}$ sensors of respective reliabilities $\mathrm{r}_{\mathrm{i}}(\mathrm{t})$ :

$R(t)=f\left(r_{1}(t), \ldots, r_{n}(t)\right)$

In the following, without loss of generality, the sensor failure rates are chosen equal. In practice, the evaluation of the system reliability uses the Mean Time To Failure function defined by the expression:

MTTF $=\int_{0}^{\infty} R(t) d t$

Let us consider the reliability of a system (fig. 1) supplied with five sensors on arcs 1, 3, 5, 6 and 7 and whose variables are linked by the two redundancy equations:

$\mathrm{q}_{1}-\mathrm{q}_{6}=0$

$\mathrm{q}_{3}-\mathrm{q}_{5}-\mathrm{q}_{7}=0$

Let us assume that measured variables are all necessary for the elaboration of the control of the system or to its supervision. That means that a failure of one or several sensors is tolerated provided that the corresponding information could be deduced from information of remaining sensors.

If the redundancy expressed in the equations (37) is not considered the probability that all sensors function, therefore that they do not fail, is:

$\mathrm{R}_{0}(\mathrm{t})=(\mathrm{r}(\mathrm{t}))^{5}=\exp (-5 \lambda \mathrm{t})$

Therefore, the MTTF of the measurement system is: 
$\mathrm{MTTF}_{0}=0.20 / \lambda$

If redundancy equations are taken into account, then some failures can be tolerated. Indeed if one of sensors intervening in the redundancy equations (37) fails, then the information that it delivers can be replaced by an estimation obtained by simple deduction. Generally, sensors whose failures are tolerated are those appearing in the redundancy equations of a system. Equations (37) show that, if a sensor fails, the value of the corresponding variable can be easily deduced from the remaining measurements. The probability that one sensor fails and that the four others well function is:

$R_{1}(t)=(1-r(t)) r(t)^{4}$

There are five possibilities of one sensor failure, the corresponding measurement being deducible.

Notice also that some failures affecting two sensors simultaneously are tolerable. Indeed, if sensors 1 and 7 fail for example, the observability of variables $1,3,5,6$ and 7 remains. For the same reason, other couples of failing sensors are acceptable: 1 and 5, 1 and 3,6 and 7, 5 and 6 and 6 and 3. Therefore, there are six possibilities involving two sensor failures, the corresponding measurements being deducible. The probability that two sensors are simulta-neously defective and that the three others function is:

$R_{2}(t)=(1-r(t))^{2} r(t)^{3}$

The case of more than two simultaneous sensor failures cannot be envisaged because some variables become unobservable. Summarising, the probability that variables $1,3,5,6$ and 7 are observable (by direct measurement or by deduction) is therefore:

$R(t)=R_{0}(t)+5 R_{1}(t)+6 R_{2}(t)$

Applying the definition (36), the global MTTF for the measurement system yields:

$\mathrm{MTTF}=0.65 / \lambda$

The comparison of the results (39) and (43) shows that a significant increasing of the measurement system reliability is achieved by taking into account the redundancy of information stemming from equations of the physical system. This simple example also points out the relationships between reliability, redundancy and observability notions. More generally, the influence of the modifications of the positions and the number of sensors on the MTTF can be studied. When several sensor configurations lead to the same degree of observability of the system, then one can choose the configuration offering the maximal MTTF. Alternately, we can define the measurement system guaranteeing a certain degree of reliability and maximizing the observability.

\section{CONCLUSION}

For the class of linear systems, we have analysed the influence of the measurement system on the state estimation of a system. This latter is especially sensitive to the number of measurements, to their distribution and to their precision. In a phase of conception, we have shown that it was possible to impose some constraints on the estimation quality and to respect them by a judicious choice of measurement system. This step can be performed on an already supplied sensor system but also on a non instrumented system. This technique could then be integrated as early as the conception phase of the process itself, the instrumentation being then part of the system. It would be suitable to extend the scope of the analysis performed to the case of process described by more complex models. Especially, the case of dynamic linear processes could be analysed by an analogous approach.

\section{REFERENCES}

Crowe, C.M., Y.A. Garcia Campos, A. Hrymak (1983). Reconciliation of process flow rates by matrix projection, part 1: the linear case. AIChE Journal , 29 (6), p. 881-888.

Crowe, C.M. (1989). Observability and redundancy of process data for steady-state reconciliation. Chemical Engineering Science, 44 (12), p. 2909-2917.

Darouach, M. (1986). Observabilité et validation des données des systèmes de grande dimension, application à l'équilibrage des bilans de mesures. Thèse de doctorat d'Etat, Nancy.

Gomolka, W., H.C. Turbatte, D. Maquin, and J. Ragot (1992). Contribution à l'analyse de l'observabilité des systèmes linéaires décrits par des équations de bilan. Revue R.A.I.R.O. A.P.I.I., 26 (2), pp. 69-89.

Kretsovalis, A., and R.S.H. Mah (1988). Observability and redundancy classification in generalized process networks. Computers and Chemical Engineering, 12 (7), p. 671-703.

Mah, R.S.H., G.M. Stanley, and D.M. Downing (1976). Reconciliation and rectification of process flow and inventory data. Industrial Engineering Chemistry Process Design Development, 15 (1), p. 175-183.

Maquin, D., M. Darouach, and J. Ragot (1989). Observability and data validation of bilinear systems. IFAC Congress, AIPAC '89, Nancy, July 3-7, 1989. In Advanced Information Processing in Automatic Control, R. Husson Ed., IFAC Symposia series, $n^{\circ}$ 5, pp. 113-118, Pergamon Press, 1990.

Ragot, J., M. Darouach, D. Maquin, and G. Bloch (1990). Validation de données et diagnostic. Traité des nouvelles technologies, série diagnostic et maintenance, Hermès, 431 pages.

Ragot, J., D. Maquin, and F. Kratz (1994). Observability and redundancy decomposition - application to diagnosis. In Advances in fault diagnosis for dynamic systems, Prentice Hall, Control Engineering Series, Chapter 13, Patton R.J., Frank P.M., Clark R.N. eds., 1994.

Romagnoli, J.A., and G. Stephanopoulos (1980). On the rectification of measurement errors for complex chemical plants. Steady-state analysis. Chemical Engineering Science, 35 (5), p. 1067-1081.

Stanley, G.M., and R.S.H. Mah (1981). Observability and redundancy classification in process networks. Chemical Engineering Science, 36 p. 1941-1954.

Turbatte, H.C., D. Maquin, C.T. Huynh, and J. Ragot (1993). Conception de l'architecture d'un système d'instrumentation - Application à un procédé pétrochimique. Tooldiag'93, International Conference on Fault Diagnosis, Toulouse, april 5-7.

Vaclavek, V. (1969). Studies on system engineering III. Optimal choice of the balance measurements in complicated chemical systems. Chemical Engineering Science, 24, p. 947.

Vaclavek, V., and M. Loucka (1976). Selection of measurements necessary to achieve multicomponent mass balances in chemical plant. Chemical Engineering Science, 31 (12), p. 1199-1205. 